\title{
Facilitating learners' web-based information problem-solving by query expansion-based concept mapping
}

\author{
Yueh-Min Huang and Ming-Chi Liu \\ National Cheng Kung University, Taiwan \\ Nian-Shing Chen \\ National Sun Yat-sen University, Taiwan
}

Kinshuk and Dunwei Wen

Athabasca University, Canada

\begin{abstract}
Web-based information problem-solving has been recognised as a critical ability for learners. However, the development of students' abilities in this area often faces several challenges, such as difficulty in building well-organised knowledge structures to support complex problems that require higher-order skills (e.g., system thinking). To resolve these issues, this study employs a semi-automatic tool that supports query expansion-based concept mapping (QECM) for assisting learners' web-based information problem-solving. The query expansion technique aims to recommend relevant concepts and linking words for building the map. The linking of concepts also uses non-taxonomic relationships for visualising a systemic model to develop complex problem-solving. An experiment was conducted by randomly dividing 50 participants into two groups, QECM (experimental) and conventional keyword-based search system, (control), to compare their performance during web-based information problem-solving tasks. The results show that the QECM system facilitated participants in extending their queries so as to enhance the comprehensiveness of their constructed concept maps. The QECM also improved the participants' information problem-solving performance by bridging concepts of an assigned task. The findings imply that learners using the QECM system can focus on the higherorder tasks of problem-solving and be better engaged in exploring real-life problems with the web.
\end{abstract}

\section{Introduction}

Information problem-solving (IPS) is an ability that is considered a critical capacity for students (Raes, Schellens, De Weyer, \& Vanderhoven, 2012). Students with better IPS skills can more effectively access and use information to find the best solution for a given problem. Thus, She et al. (2012) showed that introducing students to IPS activities could promote their creativity and aid them in constructing their own knowledge instead of relying on memorisation-based strategies. Moreover, it has been suggested that using a web-based learning context where the Internet becomes a major information source facilitates IPS instruction (Kim \& Hannafin, 2011; Raes et al., 2012; She et al., 2012). Rather than searching textbooks to find a solution, students are more engaged during web-based IPS activities where they can access realtime, multimedia resources to investigate problems in the real world (e.g., global warming). Students who are engaged in web-based IPS activities are thus motivated to actively construct knowledge instead of receiving it passively.

However, several challenges confront the development of students' web-based IPS skills because of the complicated skill sets it requires. For example, the process involves metacognitive skills such as individuals monitoring what they know and refining subsequent queries based on this (Liu, Huang, Kinshuk, \& Wen, 2013). Without adequate metacognitive skills, students tend to have a less successful performance in a web-based IPS activity (Raes et al., 2012; She et al., 2012). Research has also pointed out the influence of perceived information overload on students' cognitive processes (C. Y. Chen, Pedersen, \& Murphy, 2012), as well as the importance of scaffolding students' cognitive skills (e.g., concept map) in web-based IPS (C. Y. Chen et al., 2012; Kalyuga, Renk1, \& Paas, 2010; Raes et al., 2012).

Concept maps have been a popular tool for various pedagogical tasks (Daley \& Torre, 2010; Nesbit \& Adesope, 2006). Typical use of concept maps falls into two categories (Tseng, Chang, Rundgren, \& 
Rundgren, 2010): one is to ask learners to create a map following a specific instruction and the other is to provide learners with expert-built concept maps to scaffold learning. The former, also called concept mapping, focuses on eliciting students' acquired knowledge in order to assess their level of understanding, while the latter aims to scaffold students' learning processes using a referential knowledge structure (Ching \& Hsu, 2011). Scaffolding is often adopted when studying difficult topics, complex and ill-structured problems, or subjects dealing with a large amount of learning resources. With the help of computers, these two ways of using concept maps can be integrated to further facilitate learning for better learning outcomes (Arruarte, Elorriaga, Calvo, Larranaga, \& Rueda, 2012; Chang, Sung, \& Chen, 2001; N.-S. Chen, Teng, Lee, \& Kinshuk, 2011).

However, the current expert-skeleton concept maps used for referential or scaffolding purposes face the problem of rapidly increasing amounts of learning resources, particularly those available online. Although various computer-based tools are available to provide visual editing functions to help build expert concept maps, for example, CmapTools (Cañas et al., 2004), the actual data input still largely relies on contributions from human experts. Furthermore, Reilly (2007) noted that even when given an appropriate learning environment, students still struggle to find the core concepts and their essential connections between concepts in the welter of background information to which they are exposed. However, McMillan (2010) argued that when students are encouraged to explicitly construct and articulate connections between these concepts, knowledge transfer is enhanced. There is thus a demand for designing appropriate mechanisms by which concept maps can be constructed for scaffolding purposes, especially when it comes to learning knowledge derived from a large volume of learning resources (N.-S. Chen, Kinshuk, Wei, \& Chen, 2008).

In this work, we employ a tool with a semi-automatic mechanism to help both novices and experts retrieve online information and display the retrieved knowledge with concept map-based representations. The tool distinguishes itself from others by the support of what we will refer to as query expansion-based concept mapping, hereafter QECM. This tool can overcome the problems faced by learners with insufficient prior knowledge by presenting them with all the related concepts and linking words (Hay et al., 2008; Koc, 2012). For example, students constructing a food web for African grasslands may confine their queries to common animals, such as lions and zebras, and may not be able to retrieve all the terms that exist in this context. In addition, learners may describe similar concepts using different terms based on their individual experience. For example, some students may use the word eat in the query but the word feed may be used more often in online documents and web pages. Consequently, a large amount of relevant information may not be found due to inadequate querying of words (Hwang, Chen, Tsai, \& Tsai, 2011). With the aid of query expansion, however, information using semantically similar terms within similar sentence structures can be retrieved for learners to provide relevant and useful concepts in constructing concept maps. This mechanism has the added benefit of assisting learners to broaden the scope of their queries.

An experiment was conducted to evaluate learners' web-based information problem-solving when using two systems: the QECM system (experimental) and what we refer to herein as the conventional keywordbased search system (CKBS). In the CKBS system, students can iteratively reformulate their queries by referring to the retrieved texts for improving their searches. In this study, we attempt to answer the following question: Is the QECM system more effective than the CKBS system for students for constructing more comprehensive concept maps to benefit web-based information problem-solving?

\section{Web-based information problem-solving with concept map construction}

\section{Web-based Information problem-solving}

Web-based information problem-solving (WIPS) combines the skills needed to search and use web information to solve problems (Raes et al., 2012). Because the required skills involve the effective use of learners' sophisticated cognitive and metacognitive skills, several studies have reported on the benefits of scaffolding students' WIPS with information technology. For example, P. Kim and Olaciregui (2008) presented learning contents in a static concept map visualisation, and found the concept map group scored significantly higher in answering questions. Li and Chen (2010) introduced learners to interactive hierarchical concept maps to help students group web pages into associated topics and showed that the interactive concept maps were able to increase the efficiency in completing assigned information 
gathering tasks. M. C. Kim and Hannafin (2011) further proposed five problem-solving steps (identification \& engagement, evidence exploration, explanation reconstruction, presentation \& communication, and reflection \& negotiation) and stressed the importance of technology in scaffolding these steps. For instance, technology-enhanced scaffolding should facilitate evidence exploration by replacing lower-order tasks thereby allowing learners to allocate cognitive resources to higher-order tasks. Explanation reconstruction can be supported by connecting retrieved evidence to theories and subsequently revising naïve assumptions. Furthermore, the technology can support the step of presentation \& communication by visualising solutions and explanations, and sharing constructive feedback with peers and teachers.

Although these earlier studies found that technologies have some advantages with regard to facilitating the development of students' WIPS, such as visual representations and interactivity, the advantages may be reduced when it comes to solving complicated problems. For example, Hickey, Kindfield, Horwitz, and Christie (2003) pointed out that complex cause-and-effect problems remain challenging for many students because students require a visualised model of the problem domain that can be run forwards and backwards to generate a correct answer. In addition, Lee, Jonassen, and Teo (2011) emphasised that building systems models to represent ill-structured problems improves problem-solving. In other words, when it comes to complex information problems, students need not only to be supported with a searchassisted tool to explore web resources but also be better scaffolded with a more comprehensive knowledge structure so that they can visualise the potential solutions and can effectively reconstruct the model by comparing the potential solution with the searched results.

\section{Constructing good concept maps to facilitate information problem-solving}

According to the formal definition of concept maps (Novak \& Cañas, 2006), a relationship between two concepts should be illustrated by a line, with a linking word written on it. Two or more concepts connected by linking words, called a proposition or a semantic unit, will form a meaningful statement. However, the output of concept maps may be incomplete. Incomplete maps do not show propositions due to the problem of semantic loss during document processing.

The incomplete maps represent the structure-oriented relationships of a particular domain, such as families of animals, and this kind of concept map can only help the learner to understand the parent-child (preceding-following) relationship between two concepts. However, when it comes to the more complex knowledge structure by which students can reason, interpret, and understand, for example, the concept of a food chain in an ecosystem domain (Le Heron \& Sligo, 2005), only a much richer map containing semantic relationships between concepts can meet the demand more effectively than an incomplete one.

Therefore, instead of connecting concepts using taxonomic relationships (see Figure 1), the QECM system highlights the importance of linking concepts with non-taxonomic relationships, such as a feeding relationship (see Figure 2). Non-taxonomic relationships, which have been proven as an effective way to inspire students' problem-solving skills (Lee et al., 2011; McMillan, 2010), connect two concepts using meaningful semantic relationships. In other words, connecting concepts with non-taxonomic relationships offers learners a sequence of extensible, comprehensible paths through a complex domain of knowledge, in contrast to a taxonomic relationship that straightforwardly presents simplified relationships by a parent-child (preceding-following) relationship between concepts.

Furthermore, a teacher with a map containing well-chosen linking words can develop an instructional strategy more methodically, for example, if teaching about a food chain. Hypothetically, the teacher would first teach students about one organism, then give students another organism and eventually examine the feeding relationship between them. In the second step, the teacher would explain the definition of a food chain. In the third step, in order to help students understand the concept of a food chain, the teacher would give students many pictures of organisms and ask them to construct a simple food chain with them (e.g. grass $\rightarrow$ rabbit $\rightarrow$ fox, or grain $\rightarrow$ mouse $\rightarrow$ snake, or fish $\rightarrow$ bear). In the final step, the teacher would show students a diagram of a food web and ask them to explain the relationships of organisms in a food web. By gradually teaching about the organisms and the feeding relationships among them, students may acquire a deeper understanding of the concept of a food web in an ecosystem. The above example describes the potential benefits of representing a map containing wellchosen linking words for the QECM system. 


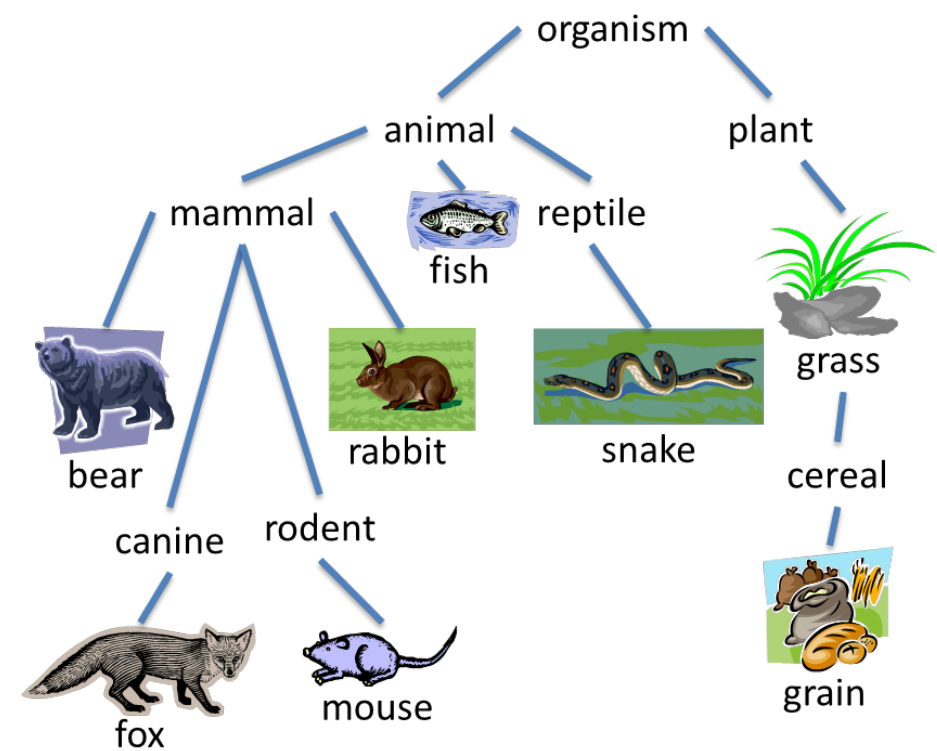

Figure 1. Example of taxonomic relationships

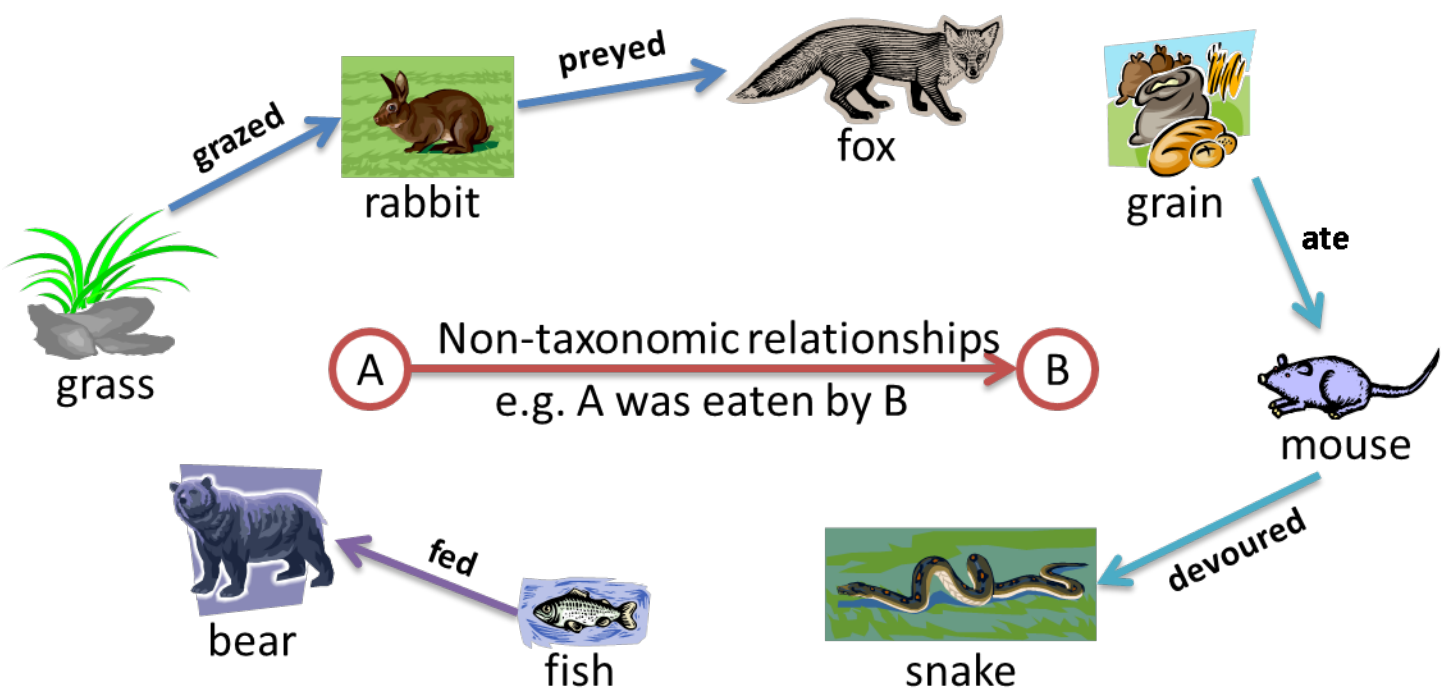

Figure 2. Example of non-taxonomic relationships

However, as pointed out by Novak and Cañas (2006), the most challenging and difficult aspect of constructing a concept map is constructing the propositions; that is, determining what linking words will clearly depict the non-taxonomic relationships between concepts. It is thus important to guide the learners to develop good maps in a disciplined way, such as starting with basic ideas and then gradually moving to more complex concept maps.

\section{Constructing concept maps with query expansion}

Learners will not always use the exact or accurate terms to search for information, especially when they have insufficient prior knowledge about the domain (Gong, Muyeba, \& Guo, 2010). In order to improve the performance of information retrieval, the search engine will expand the user's original search queries using synonyms or various morphological forms of queries. This technique is called query expansion (Gong et al., 2010). Such augmented queries can retrieve information that is more relevant and further assists the learner's search ability to use better query terms to obtain on-demand information. 
Since the QECM system employed in this work expands a learner's original query through extracting semantically similar terms within similar sentence structures, it can enable those who lack sufficient prior knowledge to discover all of the query terms for a particular topic. Even more, it can also resolve the problem of learners with varied backgrounds, who may use a linking word with a similar meaning in different terms (Y. M. Huang, Huang, \& Wu, 2014). In other words, query expansion serves to resolve problems that may arise due to the students' diverse backgrounds or gaps in knowledge in order to support their construction of concept maps.

\section{Design of the QECM system}

To efficiently and accurately extract propositions from web documents, this study proposes a framework based on syntactic analysis and semantic techniques (see Figure 3). The core processes of the framework include sentence extraction, semantic role annotation, and query expansion (see Table 1 for explanations of the terminology used in this framework). In the first stage, raw sentences containing query terms are extracted by an Internet search engine. In the second stage, the raw sentences are then parsed by semantic role annotation to extract the semantic role for each term in an individual sentence (further details of this process are presented in a later section). Then the propositions that contain meaningful structures (who did what to whom, e.g., [A cheetah $\left.{ }_{\text {who }}\right]\left[\right.$ eats $\left._{\text {did what to }}\right]\left[\right.$ an impala whom $\left._{1}\right]$ ) are collected in the database. If the extracted propositions are not enough to model complex knowledge, the process of query expansion can be used to generate more concepts or linking words. More specifically, after extracting the semantic roles of the terms in each retrieved sentence, the possible surrounding arguments of actions are extracted as candidate concepts. Those candidate concepts with semantics similar to the existing concepts are considered as expanded concepts. Moreover, the terms that appear within two concepts are extracted as the candidate linking words. Those candidate linking words with semantics similar to the existing linking words are considered as expanded linking words. In this manner, a wealth of context-relative sentences can be extracted by using these expanded words.

Table 1

Terminology used in the framework

\begin{tabular}{|c|c|c|}
\hline Terms & Definition & Example \\
\hline Action & $\begin{array}{l}\text { One instance of verbs applying to } \\
\text { something }\end{array}$ & Eat is a verb describing "take in food" \\
\hline Semantic role & $\begin{array}{l}\text { The classes of semantic relationships in } \\
\text { describing of the arguments of verbs } \\
\text { (e.g., Agent, Patient) }\end{array}$ & $\begin{array}{l}\text { The verb "eat" has two arguments } \\
\text { describing a relationship between them } \\
\text { e.g., }\left[\mathrm{A}_{\text {who }}\right][\text { eats }]\left[\mathrm{B}_{\text {whom }}\right] \text {. }\end{array}$ \\
\hline Agent & $\begin{array}{l}\text { The one that actively imposes an } \\
\text { Action to others }\end{array}$ & $\begin{array}{l}\text { "A cheetah eats an impala", "cheetah" is } \\
\text { an Agent. }\end{array}$ \\
\hline Patient & The one that has an Action applied to it & $\begin{array}{l}\text { "A cheetah eats an impala", "impala" is a } \\
\text { Patient. }\end{array}$ \\
\hline
\end{tabular}




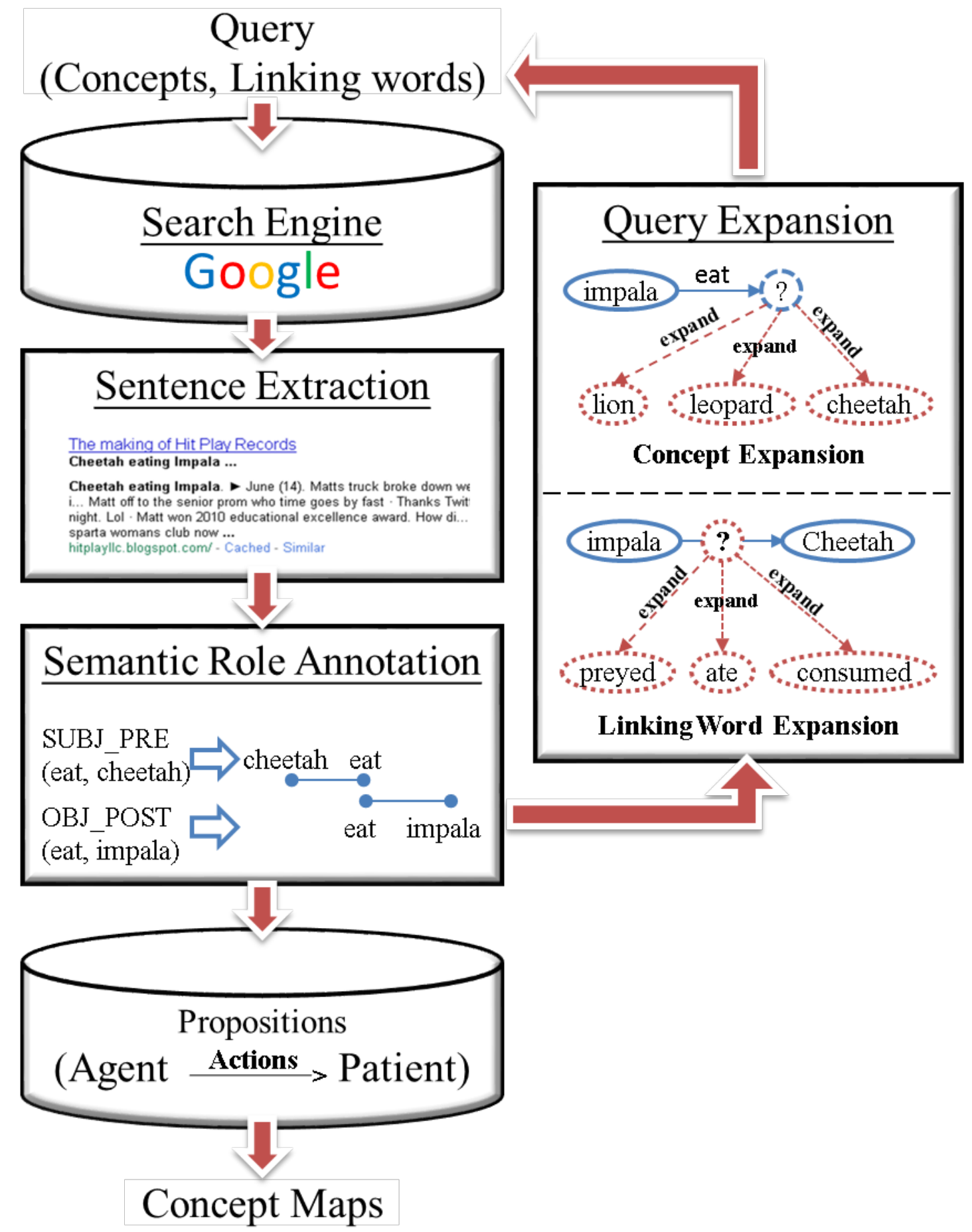

Figure 3. Framework of the concept map construction process

Figures 4 and 5 show the user interface of the QECM system and its process flow. Students can initiate a search beginning with some basic concepts and their linking words, for example, impala and cheetah as concepts and eat as the linking word. The system will then expand more concepts and linking words by using semantically similar words within similar sentence structures, for example, lion and zebra as concepts and graze and prey as linking words. In other words, presumably students have constructed the fundamental knowledge of the basic concepts and the linking words. Thus, they should be able to compare the constructed knowledge with the newly generated relevant concepts and linking words from the system and progressively select the most appropriate two concepts and one linking word, as shown in 
Figure 4. Subsequently, the system will display the number of sentences on the web containing the proposition to students as the degree of applicability. Finally, students can pick up the credible propositions to present the architecture of the constructed concept map.

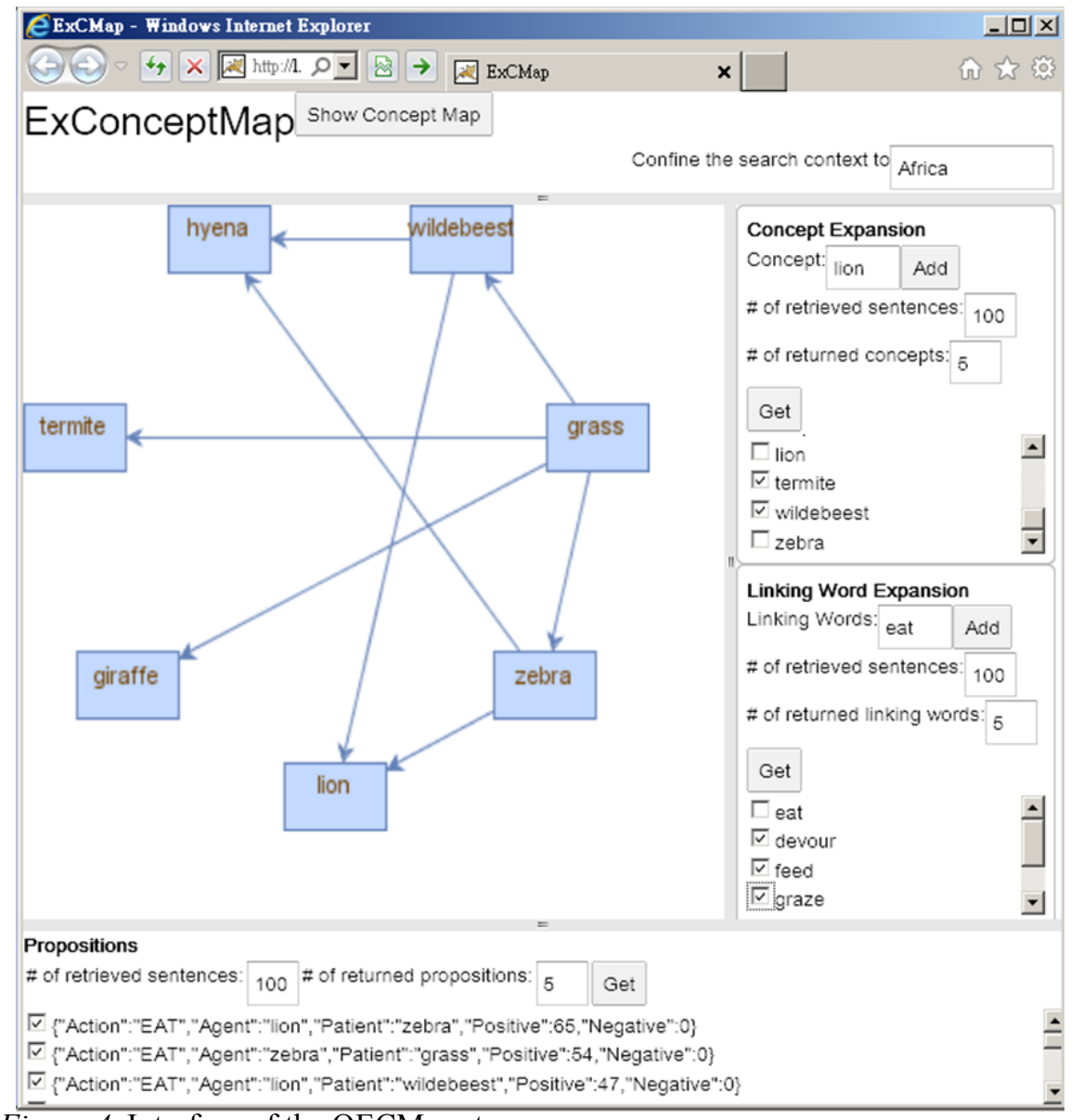

Figure 4. Interface of the QECM system

\section{Semantic role annotation}

The input in this process is a single sentence, and the output is the same sentence with the semantic roles identified. For example, the sentence "a cheetah eats an impala" is the input in this module, and the sentence "[A cheetah $\left.{ }_{\text {Agent }}\right]\left[\operatorname{eats}_{\text {Action }}\right]\left[\mathrm{an}\right.$ impala $\left.{ }_{\text {Patient }}\right]$ " is the output. Here, the word Agent refers to the participant that deliberately performs an action; and the word Patient refers to the participant being acted upon. The agent and patient are thus semantic roles related to the action. As shown in Figure 6, this process contains two steps.

Deep syntactic dependency analysis

This step reorganises a raw sentence into a set of dependencies between words, such as the subject and the object being dependents of a verb, by using a syntactic dependency parser, where a Xerox Incremental Parser (XIP) (Aït-Mokhtar, Chanod, \& Roux, 2002) is used.

Semantic dependency detection

The output of this step is the detection of semantic dependencies between words. More specifically, this step detects the sentences containing a particular structure (the agent that performs the action on the patient). This detection is based on the deep syntactic analysis outlined above, and it can be seen that these deep syntactical dependencies correspond roughly to the agent role subsumed by the SUBJ relation and to the patient role subsumed by the OBJ relation (Brun \& Hagege, 2009). The sentences were thus annotated with these semantic relationships. 

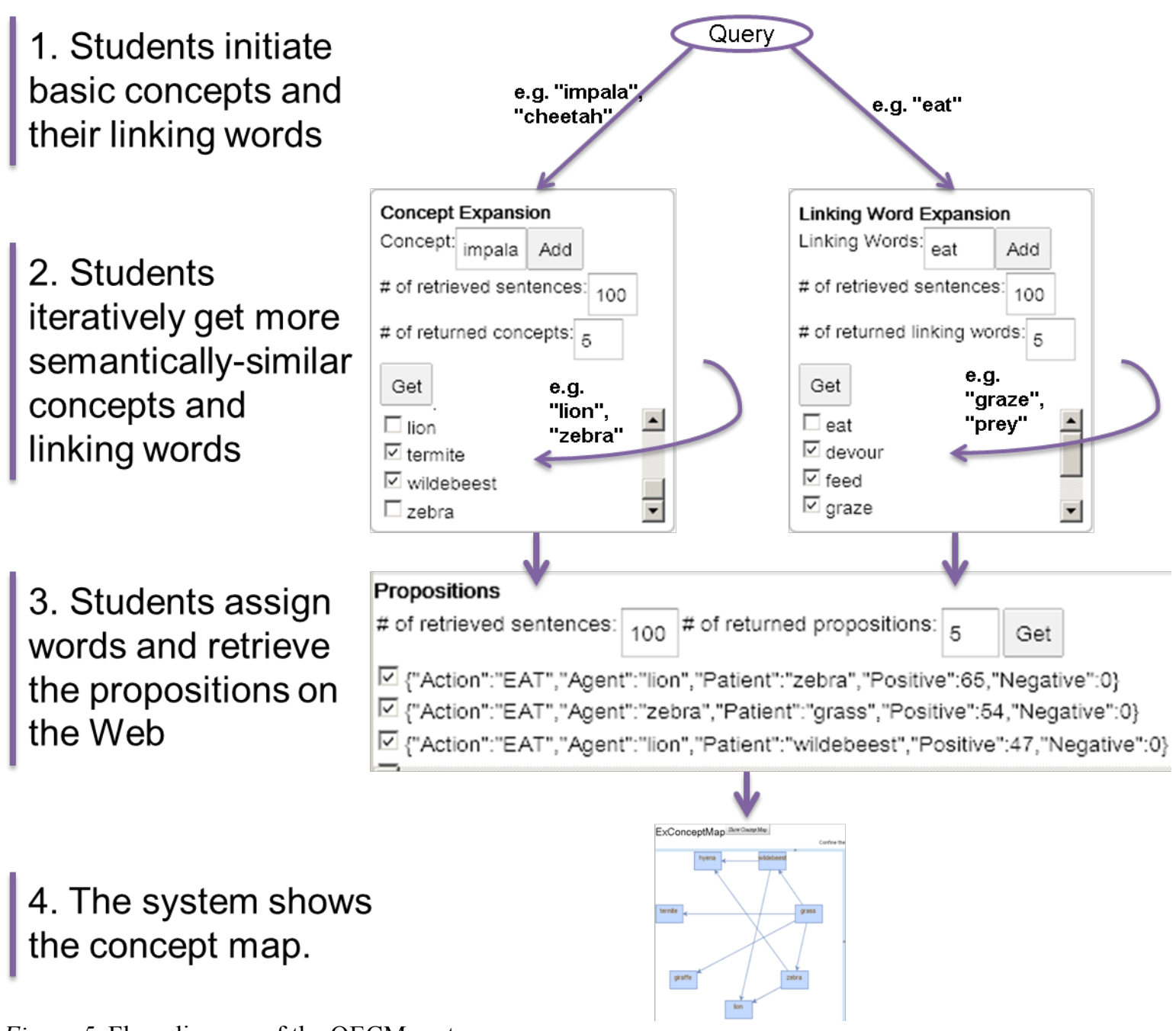

Figure 5. Flow diagram of the QECM system

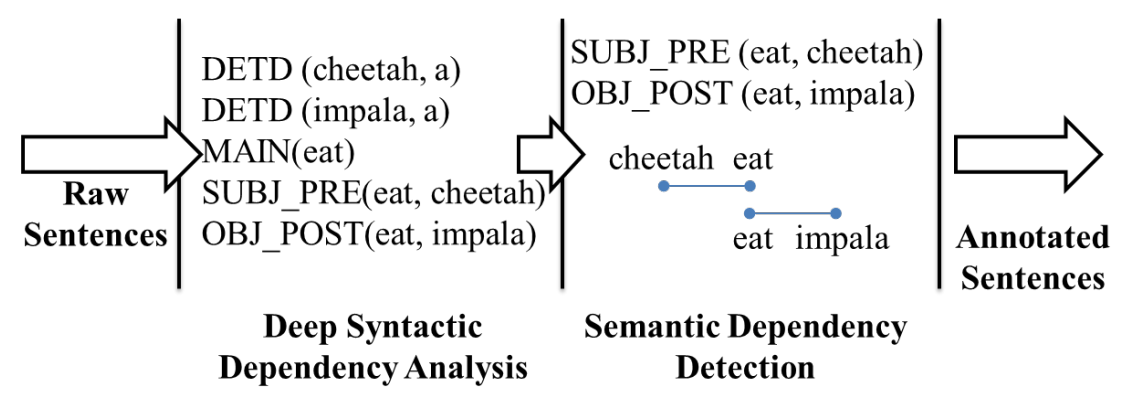

Figure 6. Semantic role annotation process

\section{Methodology}

In order to examine the students' web-based information problem-solving performance during use of the QECM system, the Google search system (CKBS system) was chosen as the counterpart, with both systems sharing the same web resources. Google's search system was considered by the students to be easier to use because it was more familiar to them. To make the comparison fair, students were asked not to use any advanced functions provided by Google. In addition, students in the CKBS group were allowed to represent the architecture of constructed concept maps. 


\section{The problem domain}

The problem domain used in the experiment was a food web pertaining to environmental protection problems, which involves a growing attention to ecological education. Although students had learned the essential notions of a food web within the context of traditional school instruction, researchers have noted that there is a deficiency of current ecological education resulting from the difficulty of applying learned scientific knowledge to daily life (Y.-M. Huang \& Chiu, 2014; Sander, Jelemenská, \& Kattmann, 2006). Moreover, knowledge of this subject is often taught piece by piece, lacking a systematic view (Grotzer \& Basca, 2003; Y. M. Huang, Liang, Su, \& Chen, 2012). Therefore, the tasks presented in this study were web-based information problems regarding species conservation. Students were first asked to search the web for examples of plants and animals with which to construct the model food web. By doing so, they could reasonably infer the keystone species that are critical to the functioning of the ecosystem to create a model food web.

\section{Participants}

The participants were 50 undergraduate students from universities in southwestern Taiwan who were recruited online and paid for their participation. None of them could correctly answer questions related to more than 2 out of 12 ecological relationships used in the assigned tasks, and this pre-test was conducted without any assistance tool. The participants were then randomly assigned to one of the two groups: the group using the QECM system (experimental) and that using the CKBS system (control).

\section{Task design}

The participants were asked to identify the possible bridging concepts in each of 12 two-step pairs (e.g., start, ALGAE; target, CRAB; mediator [not presented], SEA URCHIN; relation EAT, as shown in Figure 7). The 12 two-step pairs were constructed from four cases of food webs (Africa, Antarctica, Australia, and marine). Each case contained 3 two-step pairs, where each pair was selected based on the frequency of its occurrence in sentences found in web documents (e.g., the frequency of ALGAE-mediatorOCTOPUS, ALGAE-mediator-CRAB, CRAB-mediator-DOLPHIN were high, middle and low, respectively). The Africa case contained these two-step pairs (GRASS-mediator-LION, GRASSmediator-HYENA, and ACACIA-mediator-HYENA). The Antarctica case contained these two-step pairs (SQUID-mediator-SEAL, FISH-mediator-KILLER WHALE, and KRILL-mediator-PETREL). The Australia case contained these two-step pairs (GRASS-mediator-DINGO, TERMITE-mediator-DINGO, and CRICKET-mediator-EAGLE); The Marine case contained these two-step pairs (ALGAE-mediatorOCTOPUS, ALGAE-mediator-CRAB, and CRAB-mediator-DOLPHIN). Each task encompassed one food web case. A total of four tasks were used in the experiment.

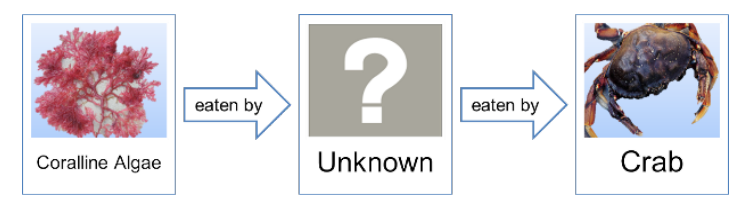

Figure 7. Example of one of the 12 two-step pairs

\section{Procedure}

First, all participants took a 20-minute training course on practising either the QECM system for the experimental group or practising searching with the CKBS system for the control group. The teaching assistant presented the participants with the importance of preserving biodiversity in environmental protection, describing the critical roles of keystone species in a food web.

Following the 20-minute computer training and introductory lesson, students were then given 80 minutes to complete the four tasks in a random order. In order to accomplish each task, they were told to begin by constructing a food web model with either the QECM or CKBS systems. Both groups were instructed to take the animals appearing in the 3 two-step pairs of each task to initiate construction of the food web model. Next, they were able to search and retrieve more relevant concepts and linking words to complete 
the food web model in each task. The participants in the experimental group were able to add some basic concepts and linking words into the QECM system. The system would then respond with more concepts and linking words that were semantically similar with the existing ones. The participants had to consider either adding more concepts and linking words or deleting the irrelevant existing ones iteratively to complete the concept map construction. On the other hand, the participants in the control group could initiate some basic concepts and linking words in the Google search engine. The search engine returned web documents containing the query words. Thus, the participants could iteratively acquire the contextrelated concepts and linking words with the web documents to complete the concept map construction.

Thirdly, the participants referred to their constructed maps to find the possible mediator in each two-step pair. Two field-related experts were invited to evaluate the accuracy of the students' results (in which each item was assigned one point, with 12 points in total for the tasks). Each item was scored one point if both experts voted that the answer was correct. Their assessments showed the Cronbach's alpha value was 0.84 , implying these evaluations were consistent.

The independent variable in this study is the system used for the web-based information problem-solving, which includes two groups: CKBS and QECM. Two dependent variables are also examined: one is the comprehensiveness of participant's concept maps, as measured by the total number of nodes and total number of linking phrases between two concepts, which was used in the study of Lee et al. (2011); the other is the participants' problem-solving performance.

\section{Results and discussion}

\section{Comprehensiveness of concept maps}

The comprehensiveness of constructed concept maps was measured by the total number of nodes and linking words participants retrieved.

Figure 8 shows the mean numbers of related concepts (corresponding to the concepts with at least one link) expanded by participants of the different groups for the four tasks. As shown in Figure 8, the QECM group retrieved more concepts than the CKBS group did in all tasks.

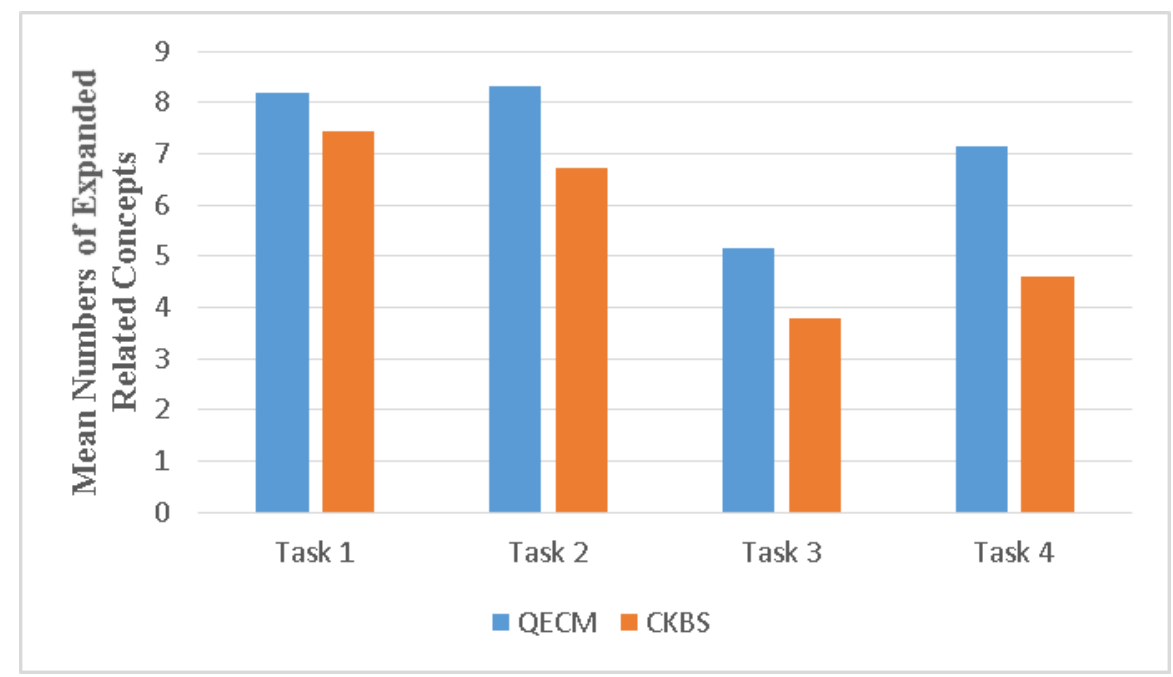

Figure 8. Mean numbers of the expanded related concepts (corresponding to the concepts with at least one link) by two groups for the four tasks

This is possibly a result of participants in the CKBS system group having to split their searches into separate queries for the two-step pairs under consideration; for example, one for lions eat *, and another for grasses are eaten by *. In comparison, the QECM system gave learners an integrated mechanism to expand their search concepts. Students could acquire the candidate mediators antelope for the two-step pairs by expanding the concepts lion and impala. This finding may imply that the function of query expansion in the QECM system could help students progressively extend their information search ability. 
Figure 9 demonstrates the participants' expanded linking words (corresponding to links with at least two concepts) in four tasks for the two groups. The results indicate that students in the QECM group could produce considerably more linking words than could those in the CKBS group. For the CKBS system, learners generally used the default linking word (eat). However, in the African food web task, the words zebra and grass are connected by the linking word graze. Although learners could acquire related linking words from the returned web documents, they may have been cognitively overloaded because of redundant information (Y. M. Huang, Huang, Liu, \& Tsai, 2013; Kalyuga et al., 2010). Moreover, they may have suffered from insufficient metacognitive strategies to appropriately reorganise their queries for retrieving the propositions (Raes et al., 2012). In contrast, the QECM system provided various expandable linking words for connecting two concepts, thus also reducing the risk of a cognitive overload.

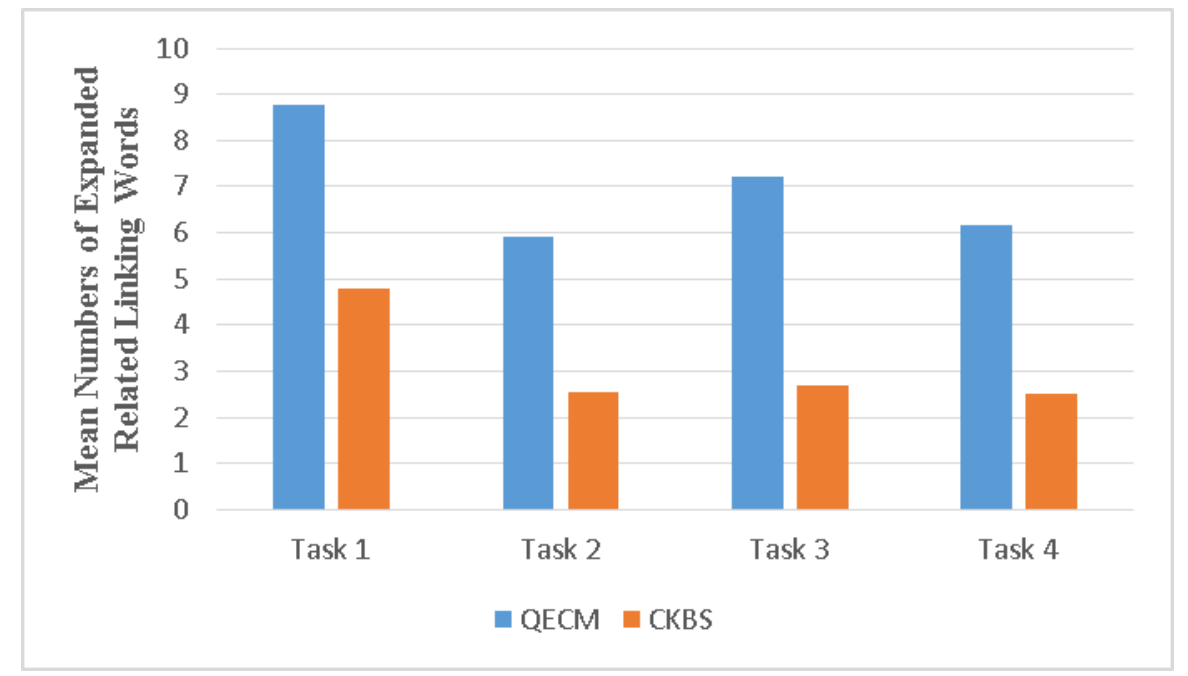

Figure 9. Mean numbers of the expanded related linking words (corresponds to links with at least two adjacent concepts) by two groups for the four tasks

Two samples of participants' constructed concept maps from two groups can further indicate the benefit of query expansion in the QECM system (see Figure 10). Figures 10(a) and 10(b) show that although the two participants in the CKBS and QECM systems were able to complete the assigned task correctly, the participant in the QECM system constructed the concept map with a more comprehensive structure. As shown in Figure 10(b), the concept map has more concepts (e.g., wildebeest) and more propositions (e.g., giraffes graze grasses, lions devour giraffes, and lions eat hyenas) than those in Figure 10(a).

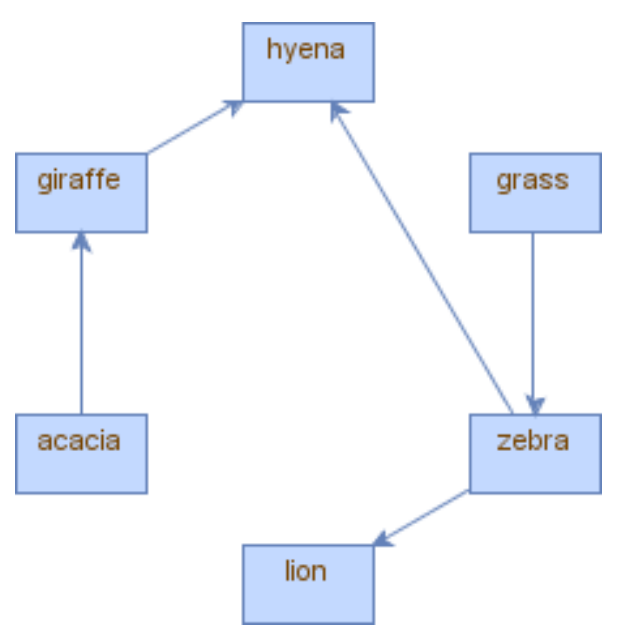

(a) The concept map from the CKBS group

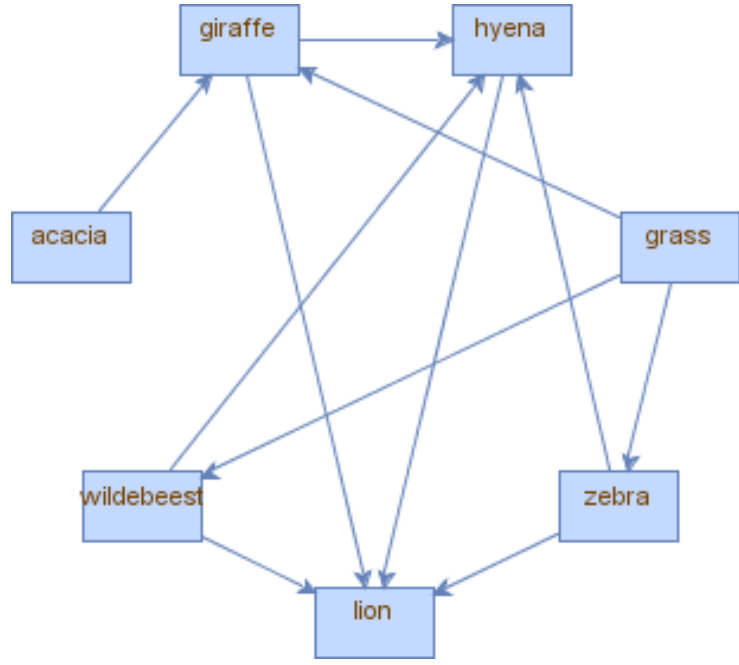

(b) The concept map from the QECM groups

Figure 10. Two samples of participants' constructed concept maps from two system groups 


\section{Problem-solving performance}

The results of the $t$ tests (see Table 2) show that participants who used the QECM system performed significantly better than those who used the CKBS system $(p<.000)$.

Table 2

Independent samples t-test for average scores on tasks in the QECM and CKBS groups $(N=50)$

\begin{tabular}{lccc}
\hline Group & Mean & $S D$ & Significance \\
\hline CKBS & 6.36 & 2.10 & $<.000$ \\
QECM & 8.88 & 1.54 & \\
\hline
\end{tabular}

This finding may be attributed to the fact that the QECM system could construct more relevant concept maps than the CKBS system. These well-organised knowledge structures allow participants to solve corresponding problems relatively effortlessly, as was observed by Kalyuga et al. (2010). M. C. Kim and Hannafin (2011) also pointed out that learners experience difficulty in attempting to solve even wellstructured problems when they lack adequate knowledge structures. However, the construction of a knowledge structure should rely heavily on participants' metacognitive skills to evaluate the accuracy of the information obtained from searching on the web (She et al., 2012). C. Y. Chen et al. (2012) further emphasised that metacognitive processes are one of the requirements for knowledge construction. Simply put, when these processes cannot function well, humans fail to construct knowledge. Therefore, the QECM system acts as a dynamic scaffold that facilitates participants' information problem-solving by extending their inquiry and enhancing the comprehensiveness of their constructed concept maps. Students with a much richer concept map were likely able to induce the plausible bridging concepts. In other words, for the participants in this study, the easier it was to construct concept maps, the more beneficial it was in facilitating their problem-solving for identifying the keystone species.

As a hypermedia learning system, QECM features learner-centric control, and thus has the potential advantages of increasing student interest and motivation, facilitating adaptive instruction, and providing affordances for active and constructive information processing (Amory, 2010; Clarebout, Horz, Schnotz, \& Elen, 2010; Hwang \& Kuo, 2011; Scheiter \& Gerjets, 2007). In general, it is thus critical that students learn how to retrieve useful information in hypermedia environments, a task that is often especially difficult when it comes to constructing referential knowledge structures. As more complex concepts and relationships are involved, building these complex knowledge structures becomes an increasingly arduous task when using the regular keyword search system (Yen, Lee, \& Chen, 2012). With the QECM tool, however, learners can not only construct more comprehensible concept maps but also expand the scope of their searches through web documents. In this way, the QECM system can serve as a web-based information problem-solving tool to generate solutions for real-life problems beyond a textbook. In the future, we will investigate other perspectives of using this tool, such as how users rate it.

\section{Pedagogical implications}

Online inquiry, where learners pose questions to investigate and access online information resources to answer those questions, is receiving increased focus in education owing to its potential to support the development of higher-order skills such as critical thinking and problem-solving (Raes et al., 2012). However, its implementation in education often faces several challenges, especially for novice learners, because the practice of online inquiry requires the effective use of learners' sophisticated cognitive and metacognitive skills (N.-S. Chen, Kinshuk, Wei, \& Liu, 2011; Liu et al., 2013; Quintana, Zhang, \& Krajcik, 2005). In particular, these skills are critical for learners to experience the complete problemsolving process, otherwise learners may be restricted in the initial stages of the problem-solving process, such as being confined to the step of evidence exploration. Their WIPS ability would be limited as well. Therefore, the proposed QECM system with embedded query expansion can contribute to scaffolding learners' metacognitive skills and enhancing their reasoning and problem-solving. In particular, the QECM system can be more useful for educating ill-structured problem-solving and science inquiry, both of which emphasise the importance of building systems models on problem-solving and conceptual change (Lee et al., 2011). Learners using the QECM system can develop the habit of building wellorganised knowledge structures for web information. The behaviour can help their WIPS and knowledge transfer. Furthermore, Brandstadter, Harms, and Grossschedl (2012) highlighted the urgency of employing highly directed and computer-based concept-mapping to understanding students' system 
thinking development in science education. The QECM system is thus better suited for conducting openended online inquiry, where learners have opportunities to be engaged in exploring real-life problems on the web.

\section{Conclusions}

In this paper, we presented the employment of a system capable of facilitating learners' web-based information problem-solving. More specifically, the proposed system first helps learners efficiently and semi-automatically build concept maps using web-based information. The constructed concept maps were used by participants as reference knowledge for solving reasoning problems, such as judging the keystone species in a food web. This system distinguishes itself from others by the support of query expansion during the construction process. The technique used in our system was able to recommend relevant concepts and linking words according to students' initial queries. We conclude that the query expansion technique facilitated learners' metacognitive skills by enhancing their accuracy of keyword evaluations.

The assigned task, with food webs as its target subject matter, included 12 two-step pairs and sought to assess participants' information problem-solving performance. Students were first asked to properly construct food webs, and then infer the bridging concept in a two-step relationship. The analysis of built concept maps reveals that participants in the QECM group used more relevant concept nodes than the CKBS group did, which echoes the feasibility of query expansion. Most notably, the QECM group was able to produce more linking words than the CKBS group. The findings may imply that the QECM system can scaffold participants' metacognitive skills to appropriately reorganise their queries for retrieving the propositions. The expansion technique improved their information problem-solving by the support of building more comprehensive concept maps.

The analysis of learners' problem-solving performance also lends support to the benefits of the QECM system. The results of the problem-solving performance show that participants who used the QECM system significantly outperformed those who used the CKBS system, and thus that in practice it can effectively improve the performance of web-based information problem-solving. The reason is that the designed problem emphasised the need of non-taxonomic relationships in constructing comprehensible concept maps, which is different from the other study (Lee et al., 2011; McMillan, 2010) that linked concepts only with a parent-child relationship. To this end, the query expansion function in the QECM system can facilitate discovering such non-taxonomic relationships and empower students in constructing semantically enriched concept maps to enhance their reasoning and problem-solving.

Finally it is worth noting that whatever subject is selected for students' concept map construction, providing students with effective tools for assisting and expanding their knowledge-building and discovery learning should be a trend. It is hoped that future work may use this tool with other databases to extend its applicability.

\section{Acknowledgments}

The authors acknowledge the support of NSERC, iCORE, Xerox, and the research-related gift funding by Mr A. Markin. This research is partially supported by the International Research-Intensive Center of Excellence Program of NTNU and the Ministry of Science and Technology, Taiwan, R.O.C., under Grant no. NSC 103-2911-I-003-301, NSC 102-3113-P-006-019-, MOST 103-2511-S-006-007-MY3, and MOST 103-2511-S-006-002-MY3.

\section{References}

Aït-Mokhtar, S., Chanod, J.-P., \& Roux, C. (2002). Robustness beyond shallowness: Incremental deep parsing. Natural Language Engineering, 8(2-3), 121-144. doi:10.1017/S1351324902002887

Amory, A. (2010). Learning to play games or playing games to learn? A health education case study with Soweto teenagers. Australasian Journal of Educational Technology, 26, 810-829. Retrieved from http://www.ascilite.org.au/ajet/submission/index.php/AJET/index

Arruarte, A., Elorriaga, J. A., Calvo, I., Larranaga, M., \& Rueda, U. (2012). Computer-based concept maps for enabling multilingual education in computer science: A Basque, English and Spanish languages case. Australasian Journal of Educational Technology, 28, 793-808. Retrieved from 
http://www.ascilite.org.au/ajet/submission/index.php/AJET/index

Brandstadter, K., Harms, U., \& Grossschedl, J. (2012). Assessing system thinking through different concept-mapping rractices. International Journal of Science Education, 34(14), 2147-2170. doi:10.1080/09500693.2012.716549

Brun, C., \& Hagege, C. (2009). Semantically-driven extraction of relations between named entities. Research in Computing Science, 41, 35-46. Retrieved from http://www.cicling.org/2009/RCS-41/

Cañas, A. J., Hill, G., Carff, R., Suri, N., Lott, J., Gómez, G., . . Carvajal, R. (2004). CmapTools: A knowledge modeling and sharing environment. In A. J. Cañas, J. D. Novak, \& F. M. González (Ed.), Concept Maps: Theory, Methodology, Technology (pp. 125-133). Universidad Pública de Navarr Press.

Chang, K. E., Sung, Y. T., \& Chen, S. F. (2001). Learning through computer-based concept mapping with scaffolding aid. Journal of Computer Assisted Learning, 17(1), 21-33. doi:10.1046/j.13652729.2001.00156.x

Chen, C. Y., Pedersen, S., \& Murphy, K. L. (2012). The influence of perceived information overload on student participation and knowledge construction in computer-mediated communication. Instructional Science, 40(2), 325-349. doi:10.1007/s11251-011-9179-0

Chen, N.-S., Kinshuk, Wei, C.-W., \& Chen, H.-J. (2008). Mining e-Learning domain concept map from academic articles. Computers \& Education, 50(3), 1009-1021. doi:10.1016/j.compedu.2006.10.001

Chen, N.-S., Kinshuk, Wei, C.-W., \& Liu, C.-C. (2011). Effects of matching teaching strategy to thinking style on learner's quality of reflection in an online learning environment. Computers and Education, $56(1), 53-64$.

Chen, N.-S., Teng, D. C.-E., Lee, C.-H., \& Kinshuk (2011). Augmenting paperbased reading activity with direct access to digital materials and scaffolded questioning. Computers \& Education, 57(2), 17051715.

Ching, Y. H., \& Hsu, Y. C. (2011). Design-grounded assessment: A framework and a case study of Web 2.0 practices in higher education. Australasian Journal of Educational Technology, 27, 781-797. Retrieved from http://www.ascilite.org.au/ajet/submission/index.php/AJET/index

Clarebout, G., Horz, H., Schnotz, W., \& Elen, J. (2010). The relation between self-regulation and the embedding of support in learning environments. Educational Technology Research and Development, 58, 573-587. doi:10.1007/s11423-009-9147-4

Daley, B. J., \& Torre, D. M. (2010). Concept maps in medical education: an analytical literature review. Medical Education, 44, 440-448. doi:10.1111/j.1365-2923.2010.03628.x

Gong, Z., Muyeba, M., \& Guo, J. (2010). Business information query expansion through semantic network. Enterprise Information Systems, 4(1), 1-22. doi:10.1080/17517570903502856

Grotzer, T. A., \& Basca, B. B. (2003). How does grasping the underlying causal structures of ecosystems impact students' understanding? Journal of Biological Education, 38(1), 16-29. doi:10.1080/00219266.2003.9655891

Hay, D. B., Kehoe, C., Miquel, M. E., Hatzipanagos, S., Kinchin, I. M., Keevil, S. F., \& Lygo-Baker, S. (2008). Measuring the quality of e-learning. British Journal of Educational Technology, 39(6), 10371056. doi:10.1111/j.1467-8535.2007.00777.x

Hickey, D. T., Kindfield, A. C. H., Horwitz, P., \& Christie, M. A. T. (2003). Integrating curriculum, instruction, assessment, and evaluation in a technology-supported genetics learning environment. American Educational Research Journal, 40(2), 495-538.

Huang, Y.-M., \& Chiu, P.-S. (2014). The effectiveness of a meaningful learning-based evaluation model for context-aware mobile learning. British Journal of Educational Technology . doi:10.1111/bjet.12147

Huang, Y. M., Huang, S. H., \& Wu, T. T. (2014). Embedding diagnostic mechanisms in a digital game for learning mathematics. Educational Technology Research and Development, 62(2), 187-207. doi:10.1007/s11423-013-9315-4

Huang, Y. M., Huang, Y. M., Liu, C. H., \& Tsai, C. C. (2013). Applying social tagging to manage cognitive load in a Web 2.0 self-learning environment. Interactive Learning Environments, 21(3), 273-289. doi:10.1080/10494820.2011.555839

Huang, Y. M., Liang, T. H., Su, Y. N., \& Chen, N. S. (2012). Empowering personalized learning with an interactive e-book learning system for elementary school students. Educational Technology Research and Development, 60(4), 703-722. doi:10.1007/s11423-012-9237-6

Hwang, G. J., Chen, C. Y., Tsai, P. S., \& Tsai, C. C. (2011). An expert system for improving web-based problem-solving ability of students. Expert Systems with Applications, 38(7), 8664-8672.

doi:10.1016/j.eswa.2011.01.072 
Hwang, G. J., \& Kuo, F. R. (2011). An information-summarising instruction strategy for improving the web-based problem solving abilities of students. Australasian Journal of Educational Technology, 27, 290-306. Retrieved from http://www.ascilite.org.au/ajet/submission/index.php/AJET/index

Kalyuga, S., Renk1, A., \& Paas, F. (2010). Facilitating flexible problem solving: A cognitive load perspective. Educational Psychology Review, 22(2), 175-186. doi:10.1007/s10648-010-9132-9

Kim, M. C., \& Hannafin, M. J. (2011). Scaffolding problem solving in technology-enhanced learning environments (TELEs): Bridging research and theory with practice. Computers \& Education, 56(2), 403-417. doi:10.1016/j.compedu.2010.08.024

Kim, P., \& Olaciregui, C. (2008). The effects of a concept map-based information display in an electronic portfolio system on information processing and retention in a fifth-grade science class covering the Earth's atmosphere. British Journal of Educational Technology, 39(4), 700-714. doi:10.1111/j.14678535.2007.00763.x

Koc, M. (2012). Pedagogical knowledge representation through concept mapping as a study and collaboration tool in teacher education. Australasian Journal of Educational Technology, 28, 656-670. Retrieved from http://www.ascilite.org.au/ajet/submission/index.php/AJET/index

Le Heron, J., \& Sligo, F. (2005). Acquisition of simple and complex knowledge; a knowledge gap perspective. Educational Technology \& Society, 8(2), 190-202.

Lee, C. B., Jonassen, D., \& Teo, T. (2011). The role of model building in problem solving and conceptual change. Interactive Learning Environments, 19(3), 247-265. doi:10.1080/10494820902850158

Li, L. Y., \& Chen, G. D. (2010). A Web browser interface to manage the searching and organizing of information on the Web by learners. Educational Technology \& Society, 13(4), 86-97.

Liu, M.-C., Huang, Y.-M., Kinshuk, \& Wen, D. (2013). Fostering learners' metacognitive skills of keyword reformulation in image seeking by location-based hierarchical navigation. Educational Technology Research and Development, 61(2), 233-254.

McMillan, W. J. (2010). Teaching for clinical reasoning - helping students make the conceptual links. Medical Teacher, 32(10), e436-e442. doi:10.3109/01421591003695303

Nesbit, J. C., \& Adesope, O. O. (2006). Learning with concept and knowledge maps: A meta-analysis. Review of Educational Research, 76(3), 413-448. doi:10.3102/00346543076003413

$\sqrt{ }$ Novak, J. D., \& Cañas, A. J. (2006). The Theory Underlying Concept Maps and How to Construct and Use Them Technical Report IHMC CmapTools 2006-01. Pensacola: Florida Institute for Human and Machine Cognition.

Quintana, C., Zhang, M. L., \& Krajcik, J. (2005). A framework for supporting metacognitive aspects of online inquiry through software-based scaffolding. Educational Psychologist, 40(4), 235-244. doi:10.1207/s15326985ep4004_5

Raes, A., Schellens, T., De Weyer, B., \& Vanderhoven, E. (2012). Scaffolding information problem solving in web-based collaborative inquiry learning. Computers \& Education, 59(1), 82-94. doi:10.1016/j.compedu.2011.11.010

Reilly, B. M. (2007). Inconvenient truths about effective clinical teaching. The Lancet, 370(9588), $705-$ 711. doi:10.1016/S0140-6736(07)61347-6

Sander, E., Jelemenská, P., \& Kattmann, U. (2006). Towards a better understanding of ecology. Journal of Biological Education, 40(3), 119-123. doi:10.1080/00219266.2006.9656028

Scheiter, K., \& Gerjets, P. (2007). Learner control in hypermedia environments. Educational Psychology Review, 19(3), 285-307. doi:10.1007/s10648-007-9046-3

She, H. C., Cheng, M. T., Li, T. W., Wang, C. Y., Chiu, H. T., Lee, P. Z., ... Chuang, M. H. (2012). Webbased undergraduate chemistry problem-solving: The interplay of task performance, domain knowledge and web-searching strategies. Computers \& Education, 59(2), 750-761. doi:10.1016/j.compedu.2012.02.005

Tseng, Y.-H., Chang, C.-Y., Rundgren, S.-N. C., \& Rundgren, C.-J. (2010). Mining concept maps from news stories for measuring civic scientific literacy in media. Computers \& Education, 55(1), 165-177. doi:10.1016/j.compedu.2010.01.002

Yen, J. C., Lee, C. Y., \& Chen, I. J. (2012). The effects of image-based concept mapping on the learning outcomes and cognitive processes of mobile learners. British Journal of Educational Technology, 43(2), 307-320. doi:10.1111/j.1467-8535.2011.01189.x 
Corresponding author: Nian-Shing Chen, nschen@mis.nsysu.edu.tw

Australasian Journal of Educational Technology (C) 2014.

Please cite as: Huang, Y-M., Liu, M-C., Chen, N-S., Kinshuk, \& Wen, D. (2014). Facilitating learners' web-based information problem-solving by query expansion-based concept mapping. Australasian Journal of Educational Technology, 30(5), 517-532. 